\title{
Assessment of Whole-Brain White Matter by DTI in Autosomal Recessive Spastic Ataxia of Charlevoix-Saguenay
}

\author{
K.K. Oguz, G. Haliloglu, C. Temucin, R. Gocmen, A.C. Has, K. Doerschner, A. Dolgun and M. Alikasifoglu
}

\begin{abstract}
BACKGROUND AND PURPOSE: Extension and characteristics of WM involvement other than the brain stem remain inadequately investigated in ARSACS. The aim of this study was to investigate whole-brain WM alterations in patients with ARSACS
\end{abstract}

MATERIALS AND METHODS: Nine Turkish unrelated patients with ARSACS and 9 sex- and age-matched healthy control participants underwent neurologic examination, molecular studies, electrophysiologic studies, and DTI of the brain. TBSS was used for whole-brain voxelwise analysis of FA, AD, RD, mean diffusivity of WM. Tractographies for the CST and TPF were also computed.

RESULTS: Molecular studies revealed 8 novel mutations ( 3 nonsense, 4 missense, and 1 frameshift insertion) and a missense variation in the SACS gene. Thick TPF displaced and compressed the CST in the pons. The TPF had increased FA, decreased RD, and increased AD, which may be attributed to hypertrophy and/or hypermyelination. Widespread decreased FA and increased RD, suggesting demyelination, was found in the limbic, commissural, and projection fibers. In addition to demyelination, CST coursing cranial and caudal to the pons also showed a marked decrease in AD, suggesting axonal degeneration. Electrophysiologic studies revealed findings that concur with demyelination and axonal involvement.

CONCLUSIONS: In addition to developmental changes of the TPF and their effects on the CST in the brain stem, axonal degeneration mainly along the pyramidal tracts and widespread demyelination in WM also occur in patients with ARSACS. Widespread tissue damage may be associated with extensive loss of sacsin protein in the brain and may explain a wide range of progressive neurologic abnormalities in patients with ARSACS.

ABBREVIATIONS: AD = axial diffusivity; ARSACS = autosomal recessive spastic ataxia of Charlevoix-Saguenay; $C S T=$ corticospinal tracts; FA $=$ fractional anisotropy; RD = radial diffusivity; TBSS = tract-based spatial statistics; TPF = transverse pontine fibers

A

RSACS (MIM 270550) is a distinctive form of hereditary, early-onset spastic ataxia, which manifests mainly by earlyonset pyramidal tract and cerebellar involvement, peripheral neuropathy, and hypermyelination of the retinal nerve fibers. Clinical variations including later-onset mental retardation, ophthalmoplegia, skeletal deformities, and biochemical abnormalities have been reported. ${ }^{1,2}$

Since the first description of patients in the CharlevoixSaguenay region of Quebec in 1978, the disease has been increas-

Received November 1, 2012; accepted after revision December 7.

From the National Magnetic Resonance Research Center (UMRAM) (K.K.O., A.C.H., K.D.), Bilkent University, Bilkent, Ankara, Turkey; and Departments of Radiology (K.K.O., R.G.), Pediatric Neurology (G.H.), Neurology (C.T.), Biostatistics (A.D.), and Medical Genetics (M.A.), Faculty of Medicine, Hacettepe University, Sihhiye, Ankara, Turkey.

Please address correspondence to Kader K. Oguz, National Magnetic Resonance Research Centre (UMRAM), Bilkent University, Cyberplaza, CBlock Level 2, BilkentAnkara, Turkey; e-mail:karlioguz@yahoo.com

三 Indicates article with supplemental on-line tables.

http://dx.doi.org/10.3174/ajnr.A3488 ingly defined worldwide, with numerous mutations and diverse phenotypes. ${ }^{2}$ The responsible gene (SACS, MIM 604490) was located on chromosome $13 \mathrm{q}$ and encodes the sacsin protein, which is widely expressed throughout the brain with a predilection for pyramidal neurons, Purkinje cells, thalamic and pontine nuclei, and reticular formation. ${ }^{3}$ Confusion with cerebral palsy and secondary spastic diplegia, and variations in the phenotype lead to underdiagnosis of ARSACS. ${ }^{4,5}$

Neuroimaging findings include slowly progressive atrophy of the superior vermis, and atrophy of the cerebellar hemispheres, cervical spinal cord, and cerebral cortex. ${ }^{5,6}$ The description of characteristic bilateral, parallel, paramedian, T2 hypointense stripes on MR imaging contributed to an increased diagnosis of ARSACS. $^{7}$

By measuring anisotropic diffusion of water in the WM, DTI detects abnormalities in the myelin, axon, or orientation of fibers within the bundle. ${ }^{8,9}$ Gazulla et al ${ }^{10,11}$ described nonquantitative abnormalities of the TPF and CST by DTI in 2 studies with 5 patients. These authors suggested a developmental basis for 
ARSACS by showing interruption of the CST in the pons by large TPF. More recently, Prodi et al ${ }^{12}$ showed involvement of several additional structures such as the forceps minor and major, superior longitudinal fasciculus, and cingulum by using an ROI approach for these predetermined areas. None of these studies investigated whole-brain WM changes in ARSACS by multiple diffusion indices.

TBSS enables an unbiased whole-brain voxelwise analysis of multi-subject FA data by using a nonlinear registration algorithm, projection of the individual data onto a mean alignment-invariant tract representation, and stringent statistical analysis. It has been increasingly used for the depiction of WM abnormalities, especially in patient groups with diseases in which alterations in WM fibers cannot be precisely predicted. ${ }^{13}$

Given the high expression of the sacsin protein in the brain, we hypothesized that WM alterations are not limited to the brain stem and the cerebellum, and TBSS would show more widespread abnormality in patients with ARSACS. We also aimed to seek electrophysiologic correlates of DTI findings.

\section{MATERIALS AND METHODS Participants}

The local institutional review board approved the study, and all participants gave written informed consent. Patients were asked to participate in this prospective study on observation of MR imaging findings and confirmation of ARSACS by genetic studies.

Nine Turkish patients with ARSACS (male/female, 6/3; age range, $5-42$ years; mean age, $23.67 \pm 13.28$ years) and 9 sex- and age-matched control participants (male/female, 6/3; age range, $5-41$ years; mean age, $23.78 \pm 12.43$ years) were included in the study.

All patients underwent neurologic and ophthalmologic examinations, molecular studies, electrophysiologic studies, structural MR imaging, and DTI of the brain.

Healthy volunteers without previous neuropsychological or systemic disease served as control participants. They had normal findings on structural MR imaging.

\section{Molecular Studies}

The method for molecular studies is given in the On-line Appendix, Part 1.

\section{Electrophysiologic Studies}

Nerve conduction studies and motor-evoked potential studies from the tibialis anterior muscle and somatosensory-evoked potential by stimulation of the posterior tibial nerve were performed. Technical details are given in the On-line Appendix, Part 2.

\section{Image Acquisition}

Imaging studies were performed on a 1.5T MR imaging scanner (Symphony TIM; Siemens, Erlangen, Germany) equipped with an 8-channel head coil. Brain MR imaging included sagittal and axial T1-weighted imaging, axial T2-weighted imaging, and T2* gradient recalled-echo. DTI applied axial single-shot echo-planar imaging (TR, $5814 \mathrm{~ms}$; TE, $98 \mathrm{~ms}$; maximal b factor, $1000 \mathrm{~s} / \mathrm{mm}^{2}$; 30 independent directions; field of view, $230 \times 230 \mathrm{~mm}$; matrix,
$128 \times 128$; number of sections, 50, with 3-mm thickness without gap).

\section{Image Processing and Analysis}

Two experienced neuroradiologists (K.K.O., R.G.) evaluated brain MR imaging for the presence of atrophy of the infratentorial structures and the cerebrum, T2 hypointense stripes in the pons, $\mathrm{T} 2$ hyperintense stripes in the lateral thalami, and susceptibility on $\mathrm{T}^{*}$ GRE images in consensus.

DTI data were analyzed by use of TBSS. All scans were corrected for head motion and eddy currents by use of the affine registration. B0 volumes of each participant were extracted and averaged. The main diffusion tensor was fitted in each voxel with the FSL DTIFit tool (http://fsl.fmrib.ox.ac.uk/fsl/fsl-4.1.9/fdt/ fdt_dtifit.html), and the FA, RD, AD, and mean diffusivity maps were calculated. After registration and alignment of individual maps to the average space as input for TBSS, the mean FA map and then the thinned mean FA skeleton (which represents the centers of all tracts common to the group) were computed. Then voxelwise statistics on $\mathrm{FA}, \mathrm{RD}, \mathrm{AD}$, and mean diffusivity were performed by use of 500 permutations. The results were corrected for multiple comparisons, and family-wise error-corrected maps at $P$ values $<.05$ were considered significant.

Because the TPF and CST in the brain stem were reported to be morphologically altered, we first recognized and evaluated these structures on a directionally encoded color FA map of each participant, and we then outlined and calculated corresponding ROIs. Supratentorial WM clusters with significant change on the resulting TBSS maps were extracted as ROIs and registered and overlaid onto an anatomic Montreal Neurological Institute template. These ROIs were labeled according to Johns Hopkins University WM tractography and the International Consortium for Brain Mapping DTI-81 WM atlases in FSL, and the mean diffusion indices of the ROIs were calculated.

3D fiber tractographies of the CST and TPF were obtained by MedINRIA (http://www-sop.inria.fr/asclepios/software/ MedINRIA). The threshold for stopping fiber propagation was FA $<0.2$ and angle $<70^{\circ}$. Seed points were located at the level of the corona radiata and medulla oblongata for the CST and central pons for the TPF.

\section{Statistical Analysis}

Statistical analyses were done with a specific software package (SPSS for Windows, version 15.0; SSPS, Chicago, Illinois). Age and sex differences between patients and control participants were tested by use of the independent-sample Student $t$ test and the Fisher exact test, respectively. The independent Student $t$ test was used for testing diffusion measures between patients and control participants. $P$ values $<.05$ were considered significant.

\section{RESULTS}

\section{Patient Demographics and Clinical Assessment}

Demographic and clinical data for patients are summarized in On-line Table 1. Delays in motor skills $(n=7)$, ataxic gait $(n=4)$, dystonia $(n=1)$, and frequent falls $(n=1)$ were presenting symptoms. Initial diagnoses of the patients were cerebral 

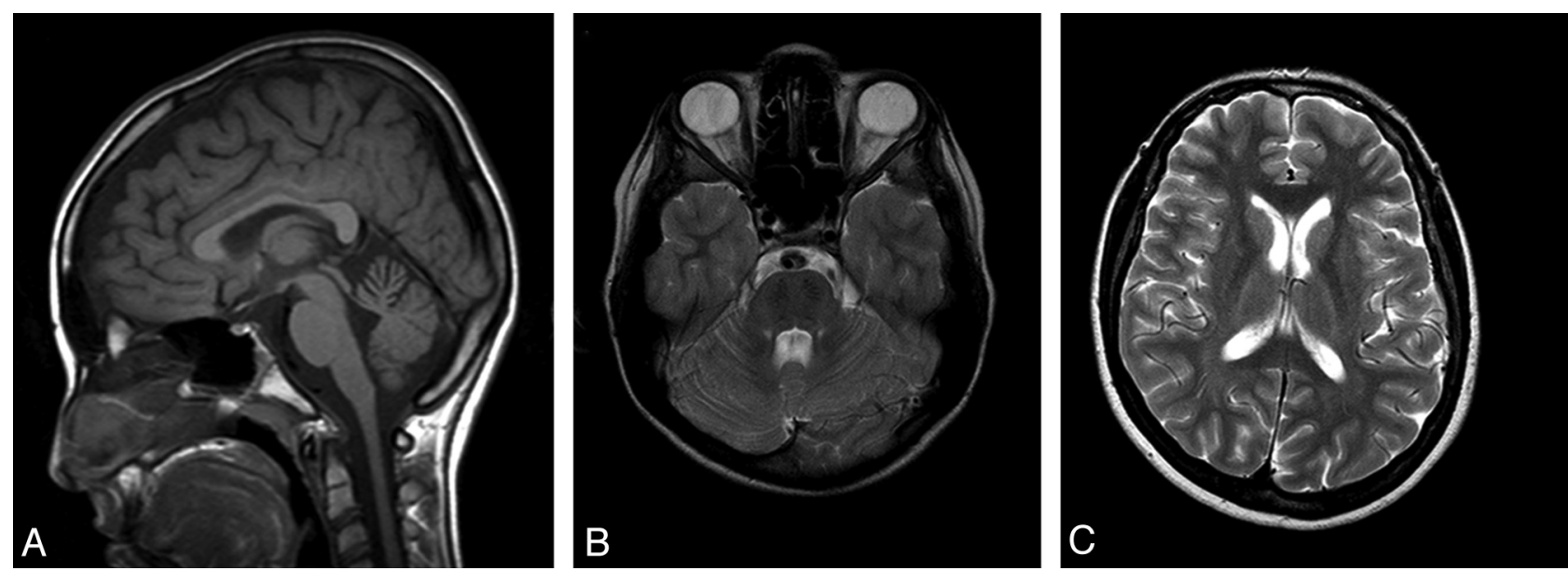

FIG 1. Sagittal T1-weighted spin-echo $(A)$, axial T2-weighted fast spin-echo $(B, C)$ images of a 14-year-old patient show atrophy of the superior vermis $(A)$ and bilateral, paramedian, T2-hypointensite stripes $(B)$ and bilateral, linear T2 hyperintensities in the lateral thalamus (C).

palsy $(n=4)$, hereditary spastic paraparesis $(n=4)$, and static cerebellar ataxia $(n=1)$. The parents were first cousins in 4 families. None of the patients had a family history of similar clinical features. Neurologic and ophthalmologic examination revealed spasticity $(n=7)$, extremity deformity $(n=2)$, cerebellar ataxia $(n=4)$, dysarthria $(n=4)$, nystagmus $(n=1)$, peripheral neuropathy $(n=7)$, and myelinated retinal fibers $(n=2)$ or thickening of the peripapillary retinal fibers $(n=1)$.

\section{Molecular Findings}

Molecular results are summarized in On-line Table 1 (detailed results in On-line Appendix, Part 3, and On-line Table 2). Eight never-reported different mutations ( 3 nonsense, 4 missense, and 1 frameshift insertion) and a missense variation were identified in 9 patients from unrelated families.

\section{Electrophysiologic Studies}

Results of electrophysiologic studies are given in On-line Table 3. Nerve conduction studies were obtained in patients; somatosensory-evoked potential and motor-evoked potential studies were obtained in 5 patients. Decreased nerve conduction velocities of the motor nerves and prolonged motor distal latencies were found in patients with ARSACS. F waves were either prolonged or absent. Sensory nerve action potentials were absent bilaterally in the sural nerves. When sensory conduction velocities could be obtained, they were reduced in the sensory nerve action potentials. Although central motor conduction times were significantly prolonged, cortical motor-evoked potential responses were remarkably low in patients without spinal and cortical somatosensoryevoked potential responses. Compound muscle action potential of the motor nerves was also low, especially in the lower extremities.

\section{Structural MR Imaging}

All patients showed superior vermian atrophy (Fig $1 A$ ) and bilateral parallel and paramedian T2-hypointense stripes in the pons (Fig 1B). Atrophy of the brain stem $(n=4)$, the cerebellar and cerebral hemispheres $(n=3)$, and bilateral T2 hyperintensities in the lateral thalami $(n=4)$ were observed (Fig $1 C)$. On T2* gradient recalled-echo imaging, no patient had susceptibility signal change.

\section{DTI}

Visual evaluation of color FA maps revealed bilaterally smaller and displaced CSTs in the pons (Fig 2A, -B) in patients with ARSACS compared with control participants. The pyramids and CSTs in the midbrain were normal in location. The pons was mostly occupied by "left-to-right" direction-coded fibers of the TPF (Fig 2A). Tractography showed thickening of the TPF and interruption of the CST in the pons with relative thinning in the midbrain and medulla oblongata in the patients (Fig 2C, $-D$ ).

The WM structures with significant change and their mean quantitative values are summarized in On-line Table 4. The TPF in patients with ARSACS had higher FA, lower RD, higher AD $(P<.05)$, and a comparable mean diffusivity $(P>.05)$ compared with control participants. In the patients, CST in the brain stem revealed significantly lower $\mathrm{FA}$, increased $\mathrm{RD}$, increased mean diffusivity $(P<.05)$, and no remarkable change in $\mathrm{AD}(P>.05)$. TBSS maps showed consistent results with these measurements of ROIs outlined from color FA maps (Fig 3).

TBSS revealed extensive reductions in FA, accompanied by increased $\mathrm{RD}$ in the supratentorial WM, including the entire corpus callosum, both cingula, fornices, superior and inferior longitudinal fasciculi, the inferior fronto-occipital fasciculi, corona radiata, internal capsules, and thalami. Bilateral superior cerebellar WM and vermis and CST in the medulla oblongata and midbrain showed a similar pattern of FA and RD changes. The extent of increased mean diffusivity and $\mathrm{AD}$ followed that of FA and $\mathrm{RD}$ changes in decreasing order. However, reduced $\mathrm{AD}$ was present along the internal capsules and midbrain, medulla oblongata, the splenium of the corpus callosum, and bilateral frontoparietal WM. No remarkable decrease in mean diffusivity was present except for a few small clusters in the subcortical temporal WM (Fig 3).

\section{DISCUSSION}

Three major results, which confirm and add to previous findings $^{10-12}$, can be derived from our data: 1) Large TPF with increased FA and $\mathrm{AD}$ and decreased RD squeezed and displaced the pontine CST with decreased FA, increased $\mathrm{RD}$, and increased mean diffusivity, as was also observed on tractography. 2) The 

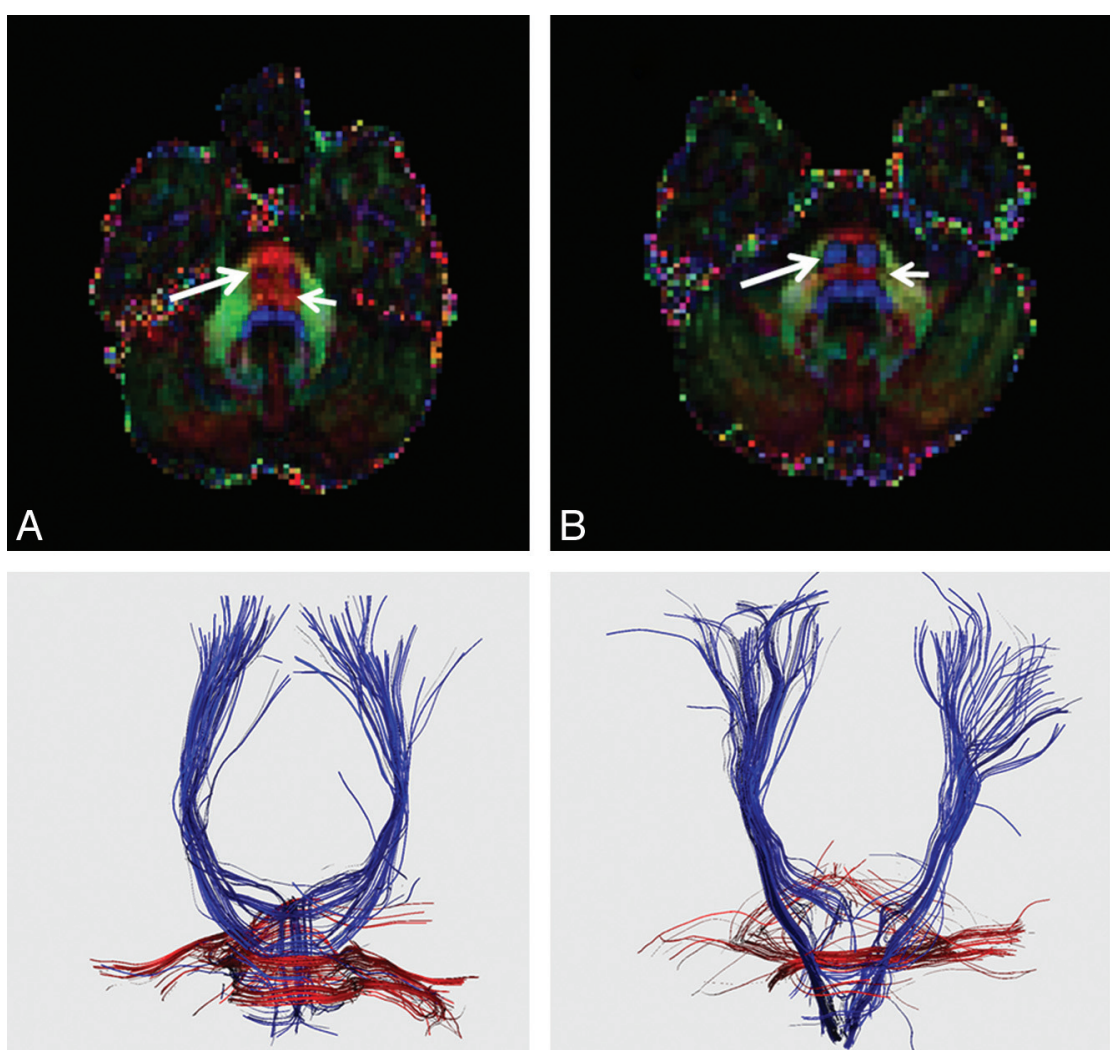

C

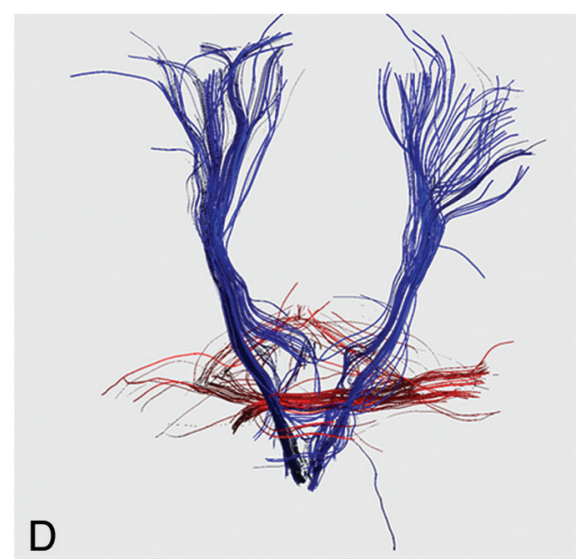

FIG 2. Directionally encoded color FA image of the same patient as in Fig $1(A)$ shows that the CST (blue, long arrow) is smaller and displaced in the pons by "left-to-right" direction-encoded TPF (red, short arrow). Tractographies of CST (blue) and TPF (red) depict interruption of CST by thick TPF $(C)$. Corresponding FA image $(B)$ and tractography $(D)$ of a sex- and age-matched control participant are also given.

CST cranial and caudal to the pons showed an apparent reduction in $\mathrm{AD}$, suggestive of axonal degeneration. 3) The supratentorial WM, including the limbic, commissural, and projection fibers, was markedly involved, as demonstrated by reduced FA, increased $\mathrm{RD}$, and increased mean diffusivity, suggestive of demyelination.

In agreement with Gazulla et al, ${ }^{10,11}$ the pontine CST was not only engulfed by the TPF (TPF was thick because of hypermyelination and/or axonal hypertrophy), but was also dislocated on directionally encoded FA images. The presence of hypermyelinated or thickened peripapillar retinal fibers has been considered a pathognomonic feature of ARSACS for quite some time. ${ }^{14} \mathrm{We}$ cannot confidently consider the thickening of the TPF and hypermyelination/peripapillary nerve thickening a result of identical pathogenesis, given the rarity of patients with abnormal fundoscopic findings in our study, similar to other non-Quebec patient populations. Furthermore, to our knowledge, no evidence has been available regarding a relationship between retinal nerve fiber layer thickening and SACS mutation and the duration and severity of ARSACS. ${ }^{15}$

$\mathrm{AD}$ (diffusion parallel to WM fibers) and $\mathrm{RD}$ (diffusion perpendicular to WM fibers) have been demonstrated to be sensitive measures in the characterization of tissue abnormalities. Experimental studies with immunohistostaining of phosphorylated neurofilament and myelin basic protein have proven that reduced
$\mathrm{AD}$ and increased $\mathrm{RD}$ correlated with axonal and myelin damage, respectively. ${ }^{16,17}$ Our finding of decreased AD in the pyramidal pathways suggests anterograde and retrograde Wallerian degeneration secondary to damage to the CST by the TPF at the pons, very similar to that observed after a pontine infarct or a spinal cord injury. ${ }^{18,19}$ However, because there was accompanying reduced FA and increased RD in the CST, one can suggest that both axonal degeneration and demyelination occur along the pyramidal tracts.

We also observed symmetric, linear, and mild T2 hyperintensities in the lateral thalami of 4 patients, which was recently suggested as reflecting degeneration of fibers of the external lamina consisting of afferent and efferent fibers between the cortex and reticular thalamic nuclei. ${ }^{12,20}$ TBSS analysis was able to detect supratentorial abnormalities beyond the thalami and motor pathways and showed widespread demyelination. Supporting our nerve conduction studies and DTI findings, previous nerve conduction studies and sural nerve biopsy examinations also revealed demyelinating neuropathy with superimposed axonal involvement in patients with ARSACS. ${ }^{11,21-23}$ Severe CST involvement, as assessed by motor-evoked potential abnormalities, was attributed to the demyelination of the CST in an autopsy study. ${ }^{24}$ Our current TBSS study, on the contrary, points out both axonal and myelin damage in the CST. These features can also contribute to differentiation of ARSACS from other degenerative diseases of mainly axonal neuropathy, such as Friedreich ataxia. ${ }^{25}$ In our study, a widespread WM abnormality is being documented first in ARSACS, in contrast to more localized involvement of WM in the superior cerebellar peduncles and peridentate area in Friedreich ataxia. ${ }^{26,27}$

Iron deposition in the basal ganglia and thalami and lipofuscin-like dens material within the lysosomes of swollen thalamic and cerebellar cortical neurons were suggested to cause T2 hypointensity in the pons and middle cerebellar peduncles..$^{5,28,29}$ Our data do not support these suggestions because of lack of paramagnetic susceptibility; T2 signal loss in the thalami or middle cerebellar peduncles; and DTI findings of material storage in swollen neurons, such as reduced mean diffusivity.

Clinical variations such as mental retardation, ophthalmoplegia, and lack of retinal nerve hypermyelination have been reported more frequently in non-Quebec patients. ${ }^{2}$ In addition to developmental abnormalities in the TPF and their mechanical effects on the CST, axonal degeneration and, more extensively, demyelination in cerebral WM may explain a wide range of neurologic abnormalities other 


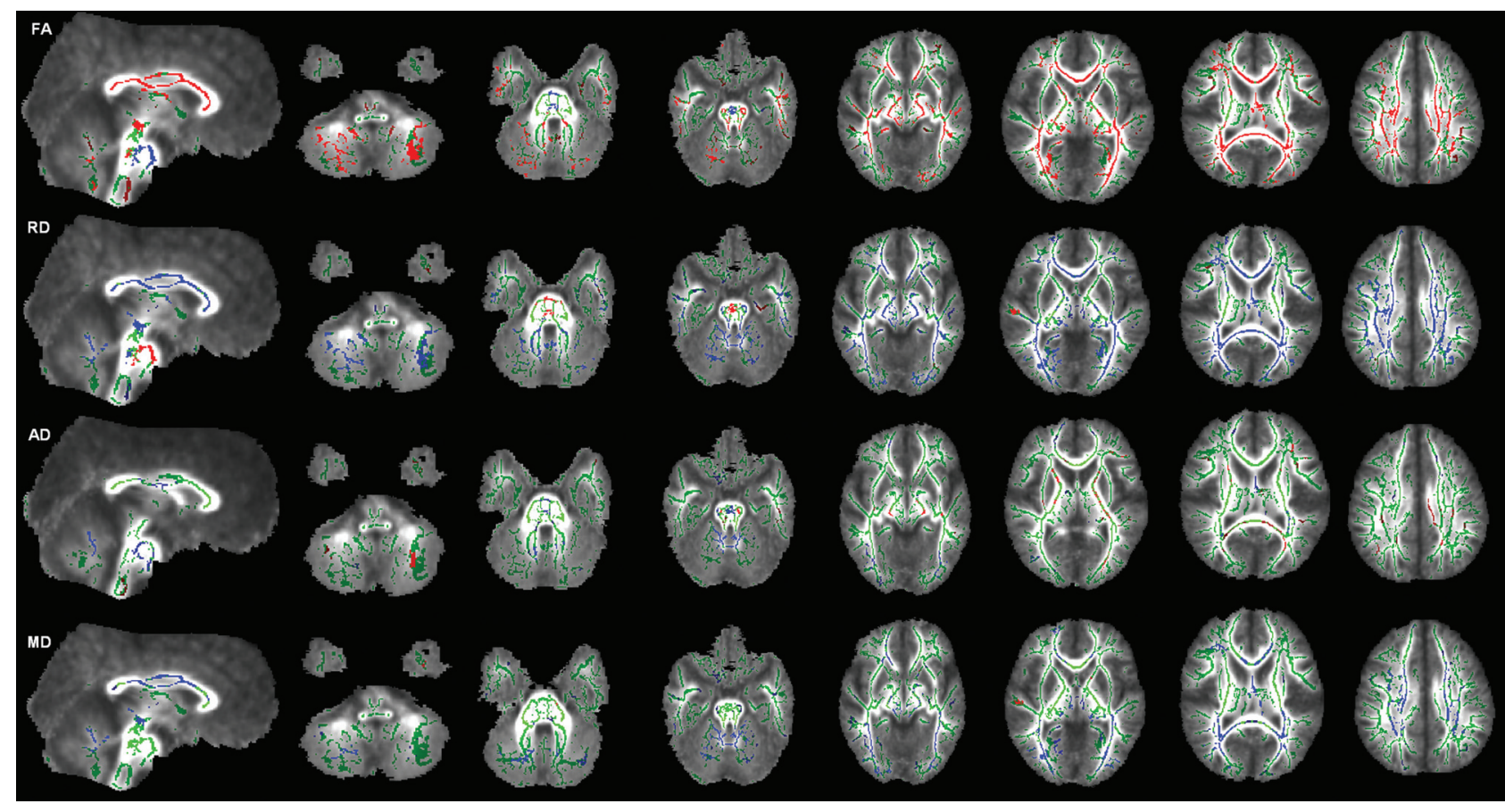

FIG 3. TBSS (family-wise error-corrected threshold-cluster extend voxel $p$ maps) display clusters with significantly different FA, RD, AD, and mean diffusivity compared with sex- and age-matched control participants at $P<.05$. For all diffusion measures, blue shows increased values and red shows decreased values. FA skeleton projected on a mean FA map is shown in green.

than spasticity and the progressive nature of the disease. Recently, Girard et $\mathrm{al}^{30}$ showed localization of sacsin to mitochondria and a cascade of detrimental effects resulting in neuronal cell death by loss of sacsin function in knockout mice. These authors further suggested the presence of some common pathophysiologic features between ARSACS and some other neurodegenerative diseases with mitochondrial impairment such as Alzheimer, Parkinson, and Huntington disease. ${ }^{30,31}$ Herein, by using TBSS, we present another common feature of ARSACS with these diseases: extensive WM alterations in the brain. ${ }^{32,33}$

Our current study was distinguished in several ways: First, to the best of our knowledge, our study was the first that investigated whole-brain WM in patients with ARSACS. Second, it quantified the abnormal pattern of diffusion indices, including $\mathrm{RD}$ and $\mathrm{AD}$, indicating widespread WM disintegration. Last, although the number of patients was limited, our patient cohort constituted the largest population of patients with ARSACS in Turkey. ${ }^{34,35}$ In our study, 8 different SACS mutations have been identified in 9 unrelated families referred to a single tertiary-care center. We believe that recognition of imaging features, as well as the identification of new mutations, will increase the rate of diagnosis of ARSACS in Turkey and other countries.

We did not measure the number of TPF and the CST because changing DTI parameters can significantly alter the results, and DTI has not been considered a precise way of measuring the length and number of fibers. ${ }^{36}$ The small CST was not found to have a significant change in the pons on TBSS maps. Because only the major WM tracts are included in the skeleton, assessment of small fiber tracts is difficult by TBSS. We overcame this problem by outlining ROIs from directionally encoded FA images after recognition of the morphologically altered structures.

\section{CONCLUSIONS}

In 9 Turkish patients with 8 new mutations, diffusion alterations suggestive of widespread demyelination and axonal involvement, mainly of the pyramidal tracts, have been demonstrated in addition to thickening of TPF and interruption of the CST in the pons. These abnormalities may reflect tissue damage related to extensive alterations in mitochondrial dynamics because of loss of sacsin function and may explain a wide range of neurologic abnormalities in patients with ARSACS.

\section{ACKNOWLEDGMENTS}

The authors thank Damagen personnel for their support in the molecular studies, Sueda Turk (Bilkent University, Department of Electrics and Electronics Engineering) for contribution in data collection, Prof. Dr. Meral Topcu (Hacettepe University, Department of Pediatric Neurology) and Prof. Dr. Ersin Tan (Hacettepe University, Department of Neurology) for their contribution of patients.

\section{REFERENCES}

1. Van de Warrenburg BP, Sinke RJ, Kremer B. Recent advances in hereditary spinocerebellar ataxias. J Neuropathol Exp Neurol 2005;64:171-80

2. Bouhlal Y, Amouri R, El Euch-Fayeche G, et al. Autosomal recessive spastic ataxia of Charlevoix-Saguenay: an overview. Parkinsonism Relat Disord 2011;17:418-22

3. Parfitt DA, Michael GJ, Vermeulen EG, et al. The ataxia protein sacsin is a functional co-chaperone that protects against polyglutamine-expanded ataxin-1. Hum Mol Genet 2009;18:1556-65

4. Bouchard JP, Barbeau A, Bouchard R, et al. Autosomal recessive spastic ataxia of Charlevoix-Saguenay. Can J Neurol Sci 1978;5:61-69

5. Bouchard JP, Richter A, Mathieu J, et al. Autosomal recessive spastic ataxia of Charlevoix-Saguenay. Neuromuscul Disord 1998;8:474-79 
6. Anheim M, Fleury M, Monga B, et al. Epidemiological, clinical, paraclinical and molecular study of a cohort of 102 patients affected with autosomal recessive progressive cerebellar ataxia from Alsace, Eastern France: implications for clinical management. Neurogenetics 2010;11:1-12

7. Martin MH, Bouchard JP, Sylvain M, et al. Autosomal recessive spastic ataxia of Charlevoix-Saguenay: a report of MR imaging in 5 patients. AJNR Am J Neuroradiol 2007;28:1606-08

8. Le Bihan D. Looking into the functional architecture of the brain with diffusion MRI. Nat Rev Neurosci 2003;4:469-80

9. Mori S, Zhang J. Principles of diffusion tensor imaging and its applications to basic neuroscience research. Neuron 2006;51:527-39

10. Gazulla J, Vela AC, Marín MA, et al. Is the ataxia of CharlevoixSaguenay a developmental disease? Med Hypotheses 2011;77:347-52

11. Gazulla J, Benavente I, Vela AC, et al. New findings in the ataxia of Charlevoix-Saguenay. J Neurol 2012;259:869-78

12. Prodi E, Grisoli M, Panzeri M, et al. Supratentorial and pontine MRI abnormalities characterize recessive spastic ataxia of CharlevoixSaguenay. A comprehensive study of an Italian series. Eur J Neurol 2013;20:138-46

13. Smith SM, Jenkinson M, Johansen-Berg H, et al. Tract-based spatial statistics: voxelwise analysis of multi-subject diffusion data. Neuroimage 2006;31:1487-505

14. Desserre J, Devos D, Sautière BG, et al. Thickening of peripapillar retinal fibers for the diagnosis of autosomal recessive spastic ataxia of Charlevoix-Saguenay. Cerebellum 2011;10:758-62

15. Vingolo EM, Di Fabio R, Salvatore S, et al. Myelinated retinal fibers in autosomal recessive spastic ataxia of Charlevoix-Saguenay. Eur J Neurol 2011;18:1187-90

16. Xie $\mathrm{M}$, Wang $\mathrm{Q}, \mathrm{Wu} \mathrm{TH}$, et al. Delayed axonal degeneration in slow Wallerian degeneration mutant mice detected using diffusion tensor imaging. Neuroscience 2011;197:339-47

17. Kim JH, Wu TH, Budde MD, et al. Noninvasive detection of brainstem and spinal cord axonal degeneration in an amyotrophic lateral sclerosis mouse model. NMR Biomed 2011;24:163-69

18. Gupta RK, Saksena S, Chandra A, et al. Retrograde Wallerian degeneration of cranial corticospinal tracts in cervical spinal cord injury patients using diffusion tensor imaging. $J$ Neurosci Res 2008;86:2271-80

19. Liang Z, Zeng J, Zhang C, et al. Longitudinal investigations on the anterograde and retrograde degeneration in the pyramidal tract following pontine infarction with diffusion tensor imaging. Cerebrovasc Dis 2008;25:209-16

20. Guillery RW, Harting JK. Structure and connections of the thalamic reticular nucleus: advancing views over half a century. J Comp Neurol 2003;463:360-71

21. Takiyama Y. Autosomal recessive spastic ataxia of CharlevoixSaguenay. Neuropathology 2006;26:368-75
22. Vermeer S, Meijer RP, Pijl BJ, et al. ARSACS in the Dutch population: a frequent cause of early-onset cerebellar ataxia. $\mathrm{Neu}$ rogenetics 2008;9:207-14

23. Peyronnard JM, Charron L, Barbeau A. The neuropathy of Charlevoix-Saguenay ataxia: an electrophysiological and pathological study. Can J Neurol Sci 1979;6:199-203

24. García A, Criscuolo C, de Michele G, et al. Neurophysiological study in a Spanish family with recessive spastic ataxia of CharlevoixSaguenay. Muscle Nerve 2008;37:107-10

25. Pandolfo M. Friedreich's ataxia: clinical aspects and pathogenesis. Semin Neurol 1999;19:311-21

26. Della Nave R, Ginestroni A, Tessa C, et al. Brain white matter tracts degeneration in Friedreich ataxia. An in vivo MRI study using tractbased spatial statistics and voxel-based morphometry. Neuroimage 2008;40:19-25

27. Pagani E, Ginestroni A, Della Nave R, et al. Assessment of brain white matter fiber bundle atrophy in patients with Friedreich ataxia. $R a$ diology 2010;255:882-89

28. Melberg A, Dahl N, Hetta J, et al. Neuroimaging study in autosomal dominant cerebellar ataxia, deafness, and narcolepsy. Neurology 1999;53:2190-92

29. Shimazaki H, Takiyama Y, Honda J, et al. Middle cerebellar peduncles and pontine T2 hypointensities in ARSACS. J Neuroimaging 2013;23:82-85

30. Girard M, Larivière R, Parfitt DA, et al. Mitochondrial dysfunction and Purkinje cell loss in autosomal recessive spastic ataxia of Charlevoix-Saguenay (ARSACS). Proc Natl Acad Sci U S A 2012;109:1661-66

31. Johri A, Beal MF. Mitochondrial dysfunction in neurodegenerative diseases. J Pharmacol Exp Ther 2012;342:619-30

32. Bohanna I, Georgiou-Karistianis N, Sritharan A, et al. Diffusion tensor imaging in Huntington's disease reveals distinct patterns of white matter degeneration associated with motor and cognitive deficits. Brain Imaging Behav 2011;5:171-80

33. Stebbins GT, Murphy CM. Diffusion tensor imaging in Alzheimer's disease and mild cognitive impairment. Behav Neurol 2009;21:39-49

34. Richter AM, Ozgul RK, Poisson VC, et al. Private SACS mutations in autosomal recessive spastic ataxia of Charlevoix-Saguenay (ARSACS) families from Turkey. Neurogenetics 2004;5:165-70

35. Gücüyener K, Ozgül K, Paternotte C, et al. Autosomal recessive spastic ataxia of Charlevoix-Saguenay in two unrelated Turkish families. Neuropediatrics 2001;32:142-46

36. Mukherjee P, Chung SW, Berman JI, et al. Diffusion tensor MR imaging and fiber tractography: technical considerations. AJNR Am J Neuroradiol 2008;29:843-52 